\title{
LRRC46 is a differentially expressed gene in brain metastatic human breast cancer.
}

Shahan Mamoor, MS ${ }^{1}$

${ }^{1}$ shahanmamoor@gmail.com

Metastasis to the brain is a clinical problem in patients with breast cancer ${ }^{1-3}$. We mined published microarray data ${ }^{4,5}$ to compare primary and metastatic tumor transcriptomes for the discovery of genes associated with brain metastasis in humans with metastatic breast cancer. We found that leucine rich repeat containing 46, encoded by LRRC46, was among the genes whose expression was most different in the brain metastases of patients with metastatic breast cancer as compared to primary tumors of the breast. LRRC46 mRNA was present at increased quantities in brain metastatic tissues as compared to primary tumors of the breast. Expression of LRRC46 in tumors was significantly correlated with patient distant metastasis-free and overall survival. Modulation of LRRC46 expression may be relevant to the biology by which tumor cells metastasize from the breast to the brain in humans with metastatic breast cancer.

Keywords: breast cancer, metastasis, brain metastases, central nervous system metastases, leucine rich repeat containing 46, LRRC46, systems biology of breast cancer, targeted therapeutics in breast cancer. 
One report described a $34 \%$ incidence of central nervous system metastases in patients

\section{Methods}

We used datasets GSE $10893^{4}$ and GSE43837 $7^{5}$ for this global differential gene expression analysis of brain metastatic breast cancer in conjunction with GEO2R. GSE10893 was generated using Agilent-011521 Human 1A Microarray G4110A technology with $n=11$ primary breast tumors and $n=3$ brain metastases from patients with breast cancer; analysis was performed using platform GPL885. GSE43837 was generated using Affymetrix Human X3P Array technology with $n=19$ primary tumors of the breast and $n=19$ brain metastases from patients with breast cancer; analysis was performed using platform GPL6480. The Benjamini and Hochberg method of $p$-value adjustment was used for ranking of differential expression but raw $p$-values were used to assess statistical significance of global differential expression. Log-transformation of data was auto-detected, and the NCBI generated category of platform annotation was used. A statistical test was performed to evaluate whether LRRC46 gene expression was significantly different between primary tumors of the breast and brain metastases in humans with breast cancer using a two-tailed t-test. For Kaplan-Meier survival analysis, we used the Kaplan-Meier plotter online tool $^{7}$ for correlation of LRRC46 mRNA expression levels with distant metastasis-free survival in $n=664$ breast cancer patients and overall survival in $n=626$ patients.

\section{$\underline{\text { Results }}$}

We performed global comparative transcriptome analysis of metastatic tumor tissues of patients with metastatic breast cancer using published microarray data ${ }^{4,5}$ to describe the transcriptional landscape of brain metastasis in human breast cancer in an unbiased fashion and for the discovery of novel therapeutic targets.

\section{LRRC46 is differentially expressed in the brain metastases of patients with brain metastatic breast cancer.}

Through blind, systems-level analysis of published microarray data ${ }^{4}$, we identified leucine rich repeat containing 46, encoded by LRRC46, as a differentially expressed gene in the breast metastatic tissues of humans with breast cancer (Table 1). When sorting each of the genes expressed in brain metastases based on significance of difference as compared to primary tumors 
of the breast in patients with breast cancer, LRRC46 ranked 55 out of 17418 total transcripts (Chart 1), equating to $99.7 \%$ differential expression. Differential expression of LRRC46 in the brain metastases of patients with metastatic breast cancer was statistically significant (Chart 1; $p=1.01 \mathrm{E}-04)$.

To attempt to validate differential transcriptome-wide differential expression of LRRC46 in brain metastases in human metastatic breast cancer, we queried a second microarray dataset ${ }^{5}$; again, we identified LRRC46 as a differentially expressed gene in the brain metastatic tissues of patients with breast cancer (Chart 2). When sorting each of the genes expressed in brain metastases based on significance of difference as compared to primary tumors of the breast, LRRC46 ranked 12099 out of 61359 total transcripts (Chart 2), equating to 80.3\% differential expression. Differential expression of LRRC46 in the brain metastases of patients with metastatic breast cancer was not deemed statistically significant (Chart $2 ; p=0.10698325$ ). Thus, transcriptome-wide differential expression of LRRC46 in the brain metastases of women with metastatic breast cancer was conserved across two independent microarray datasets, indicating that this may likely be a bona fide biological phenomena associated with metastatic breast cancer in humans.

\section{LRRC46 is expressed at higher levels in the brain metastases of patients with metastatic breast cancer.}

We obtained exact mRNA expression levels for LRRC46, in primary tumors of the breast and in brain metastasis of patients with brain metastatic breast cancer to determine direction and statistical significance of change in LRRC46 expression in brain metastatic tissues. LRRC46 was expressed at higher levels in the brain metastases of patients with breast cancer as compared to primary tumors of the breast, and this difference was statistically significant (Figure 1; $p<0.00001)$.

\section{LRRC46 expression is significantly correlated with survival outcomes in human breast cancer.}

We performed Kaplan-Meier survival analysis ${ }^{8}$ in 626 breast cancer patients in total, to evaluate whether LRRC46 tumor expression was correlated with survival outcomes in breast cancer. We observed a statistically significant correlation between primary tumor expression of LRRC46 and overall survival (OS) in patients with breast cancer (Figure 2). Patients whose primary tumors expressed low levels of LRRC46 possessed median OS of 63.36 months, while patients whose tumors expressed high levels of LRRC46 possessed median OS of 121.2 months. This difference in OS based on LRRC46 tumor expression in patients with breast cancer was statistically significant (Figure 2, Chart 3; logrank $p$-value: 0.003; hazard ratio: 0.62 (0.45-0.85)). We also observed a statistically significant correlation between distant metastasis-free survival and primary tumor expression of LRRC46 in 664 breast cancer patients (Figure 3; logrank p-value: 0.0054 ; hazard ratio: $0.63(0.45-0.87)$.

Thus, by mining published microarray data ${ }^{4,5}$ in an unbiased and systematic fashion, we identified leucine rich repeat containing 46, encoded by LRRC46, as among the genes whose expression was most different, transcriptome-wide, in the brain metastases of patients with breast cancer when compared to primary tumors of the breast; we observed significantly increased 


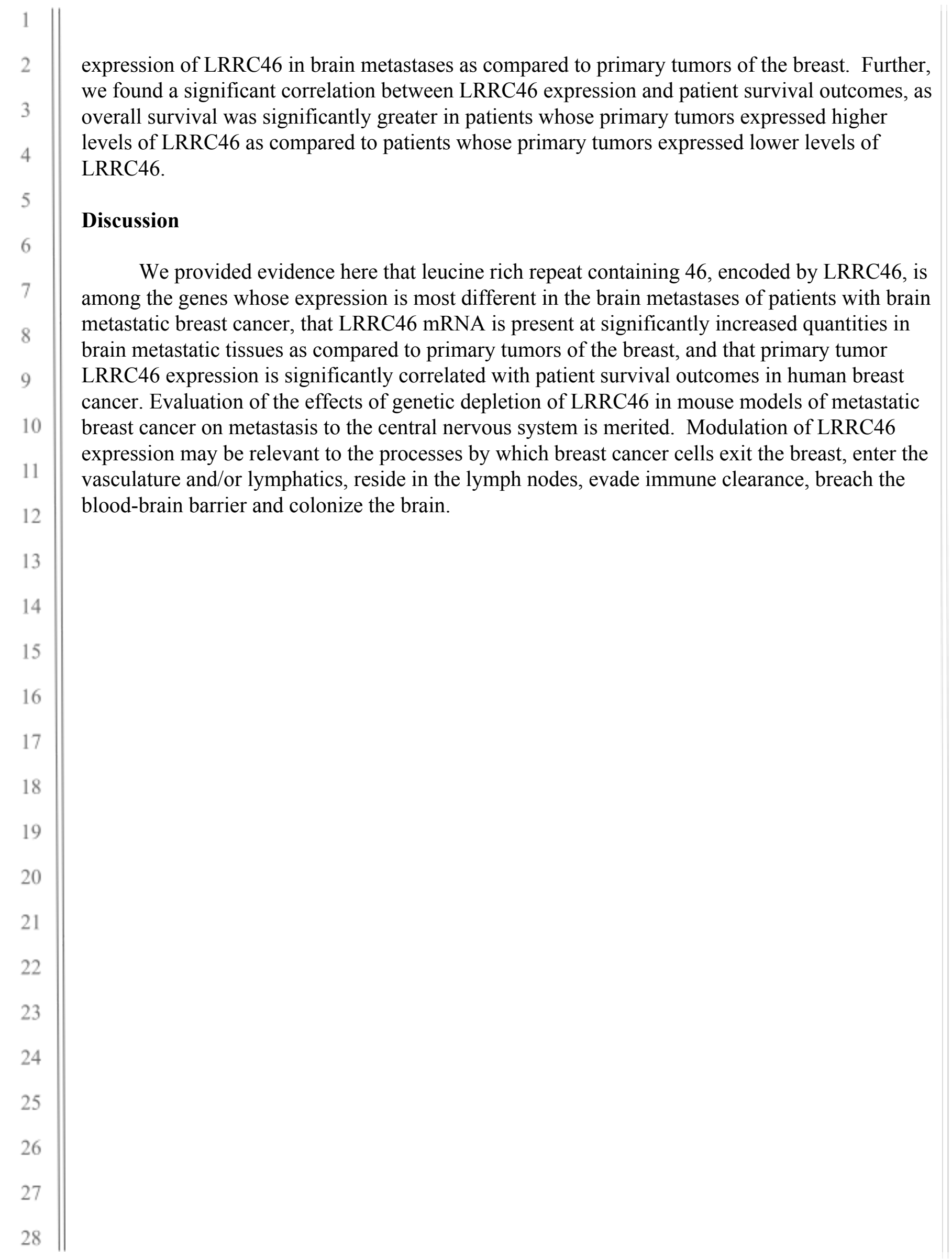




\section{References}

1. Lin, N.U., Amiri-Kordestani, L., Palmieri, D., Liewehr, D.J. and Steeg, P.S., 2013. CNS metastases in breast cancer: old challenge, new frontiers.

2. Bendell, J.C., Domchek, S.M., Burstein, H.J., Harris, L., Younger, J., Kuter, I., Bunnell, C., Rue, M., Gelman, R. and Winer, E., 2003. Central nervous system metastases in women who receive trastuzumab-based therapy for metastatic breast carcinoma. Cancer, 97(12), pp.2972-2977.

3. Tsukada, Y., Fouad, A., Pickren, J.W. and Lane, W.W., 1983. Central nervous system metastasis from breast carcinoma autopsy study. Cancer, 52(12), pp.2349-2354.

4. Weigman, V.J., Chao, H.H., Shabalin, A.A., He, X., Parker, J.S., Nordgard, S.H., Grushko, T., Huo, D., Nwachukwu, C., Nobel, A. and Kristensen, V.N., 2012. Basal-like Breast cancer DNA copy number losses identify genes involved in genomic instability, response to therapy, and patient survival. Breast cancer research and treatment, 133(3), pp.865-880.

5. McMullin, R.P., Wittner, B.S., Yang, C., Denton-Schneider, B.R., Hicks, D., Singavarapu, R., Moulis, S., Lee, J., Akbari, M.R., Narod, S.A. and Aldape, K.D., 2014. A BRCA1 deficient-like signature is enriched in breast cancer brain metastases and predicts DNA damage-induced poly (ADP-ribose) polymerase inhibitor sensitivity. Breast Cancer Research, 16(2), pp.1-10.

6. Awada, A., Colomer, R., Inoue, K., Bondarenko, I., Badwe, R.A., Demetriou, G., Lee, S.C., Mehta, A.O., Kim, S.B., Bachelot, T. and Goswami, C., 2016. Neratinib plus paclitaxel vs trastuzumab plus paclitaxel in previously untreated metastatic ERBB2-positive breast cancer: the NEfERT-T randomized clinical trial. JAMA oncology, 2(12), pp.1557-1564.

7. Györffy, B., Lanczky, A., Eklund, A.C., Denkert, C., Budczies, J., Li, Q. and Szallasi, Z., 2010. An online survival analysis tool to rapidly assess the effect of 22,277 genes on breast cancer prognosis using microarray data of 1,809 patients. Breast cancer research and treatment, 123(3), pp.725-731. 


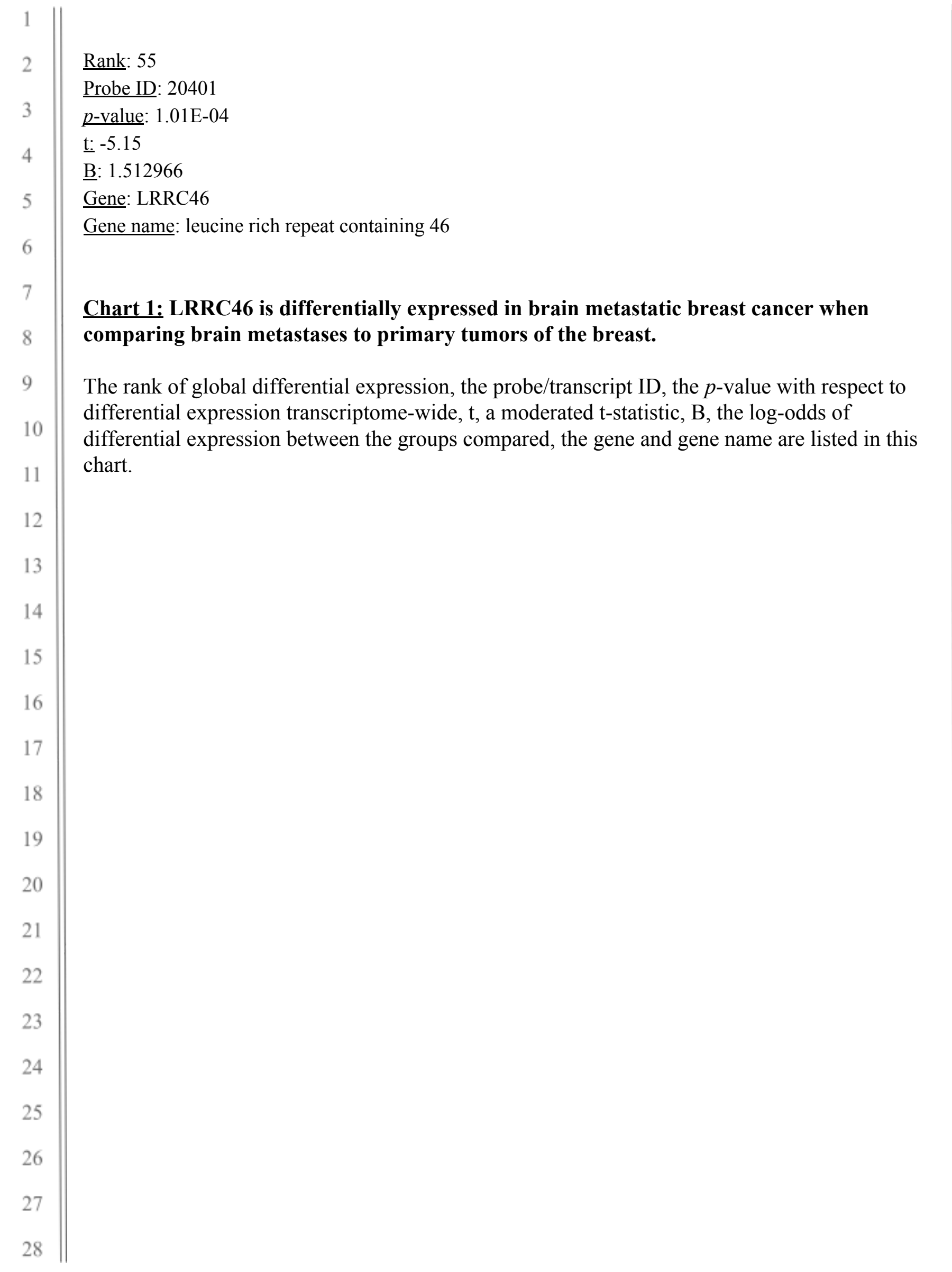




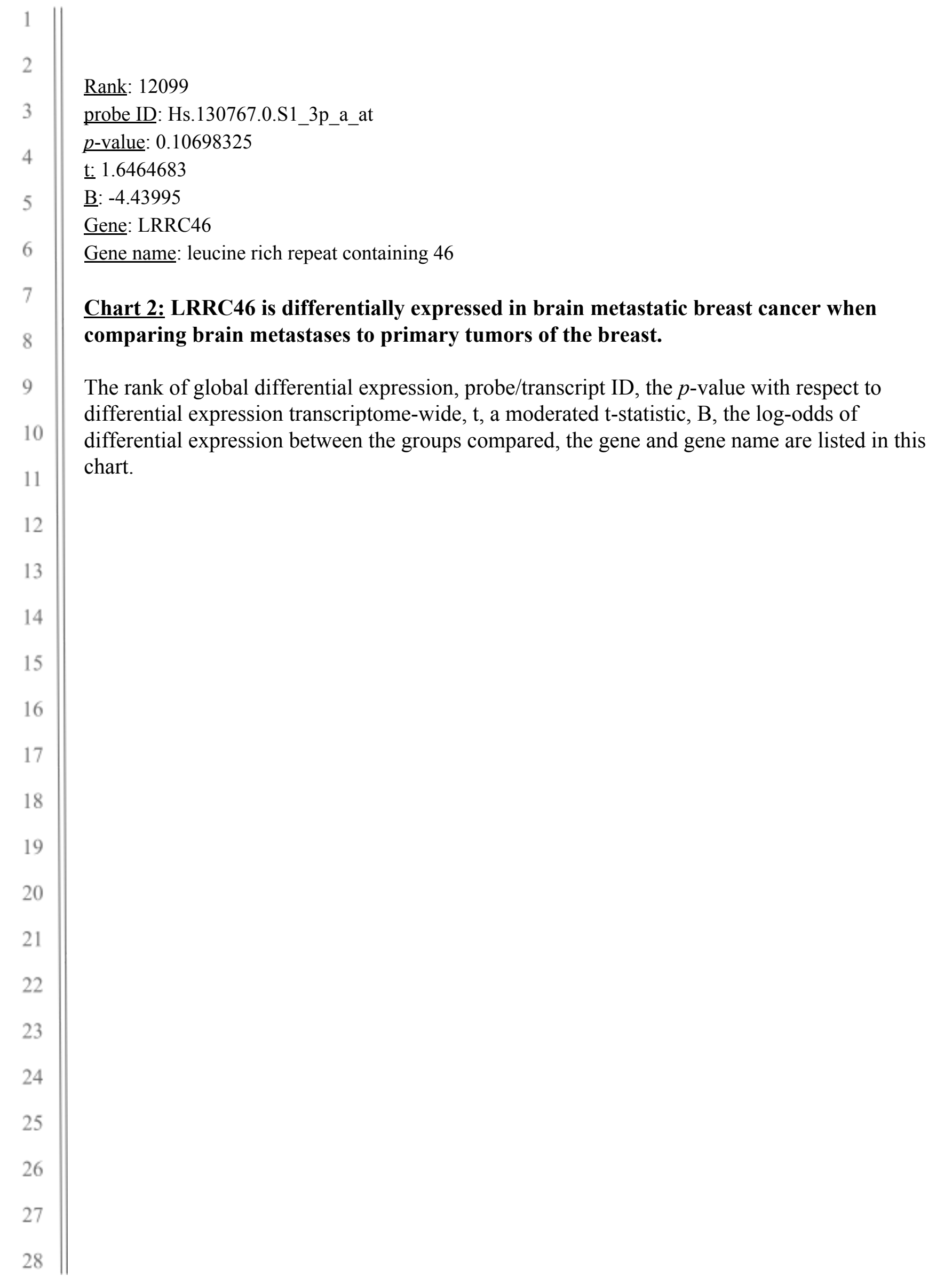




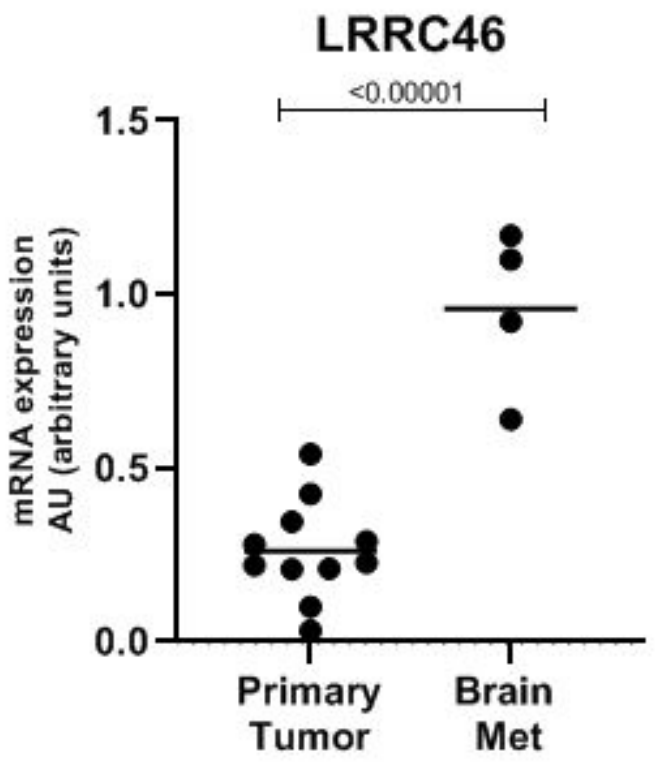

Figure 1: LRRC46 is expressed at significantly higher levels in the brain metastases of patients with metastatic breast cancer when compared to primary tumors of the breast.

The mRNA expression level of LRRC46 in primary tumors of the breast (left) and in brain metastases of women with metastatic breast cancer (right) is graphically depicted; the result of a statistical test evaluating significance of difference in LRRC46 expression between primary tumors of the breast and brain metastases is $p<0.00001$. 


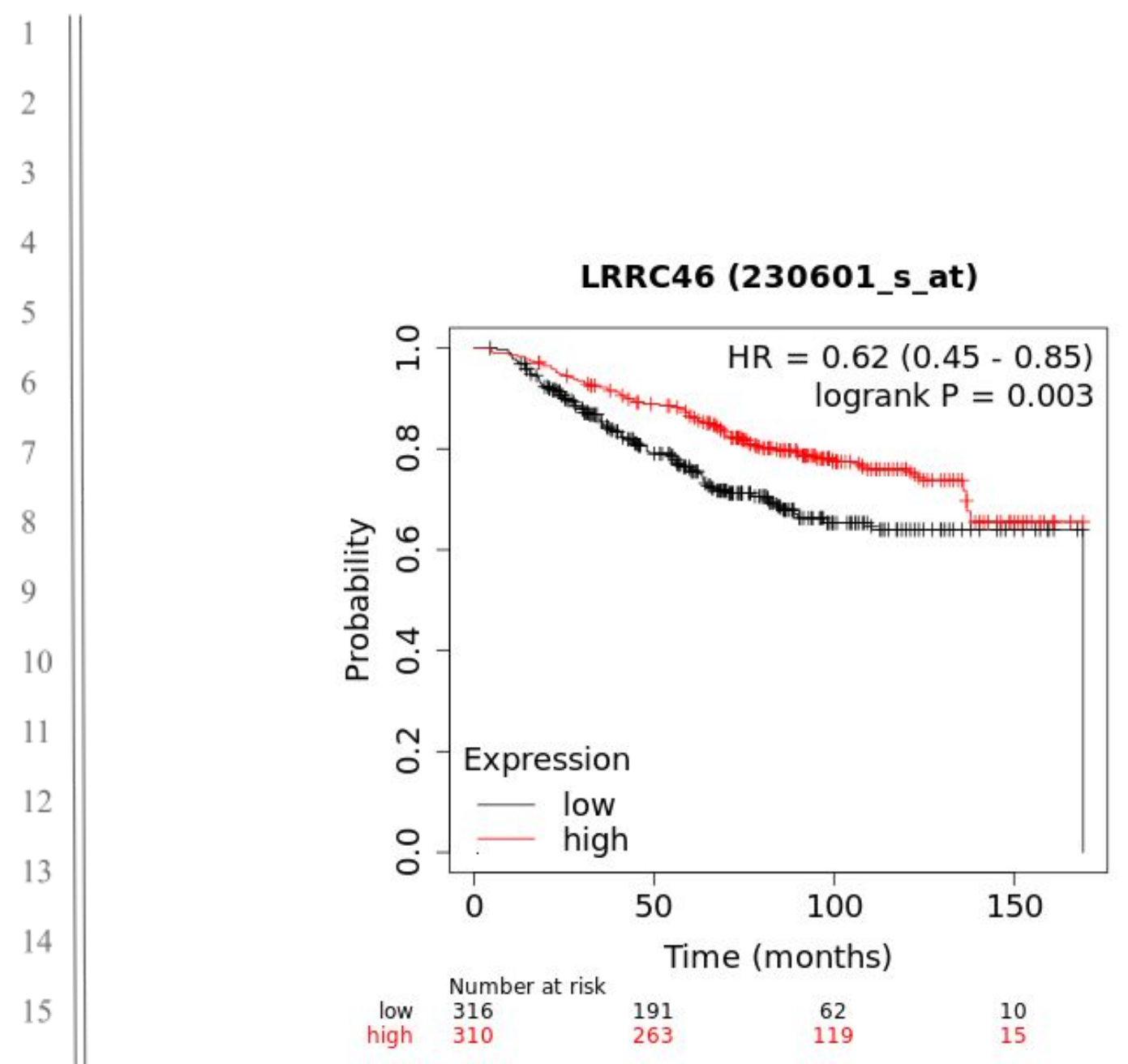

Figure 2: Significant correlation between LRRC46 primary tumor expression and overall survival in patients with breast cancer.

Depicted in this Kaplan-Meier plot is the probability of overall survival for $n=626$ total patients stratified into two groups, based on low or high expression of LRRC46 in patient primary tumors. The log rank $p$-value denoting statistical significance of difference in overall survival when comparing the two groups, as well as hazard ratio for this comparison is listed above. Listed below is the number of patients at risk (number of patients alive) per interval, after stratification based on LRRC46 expression; in the first interval, number at risk is number of patients alive; in each subsequent interval, number at risk is the number at risk less those who have expired or are censored. 


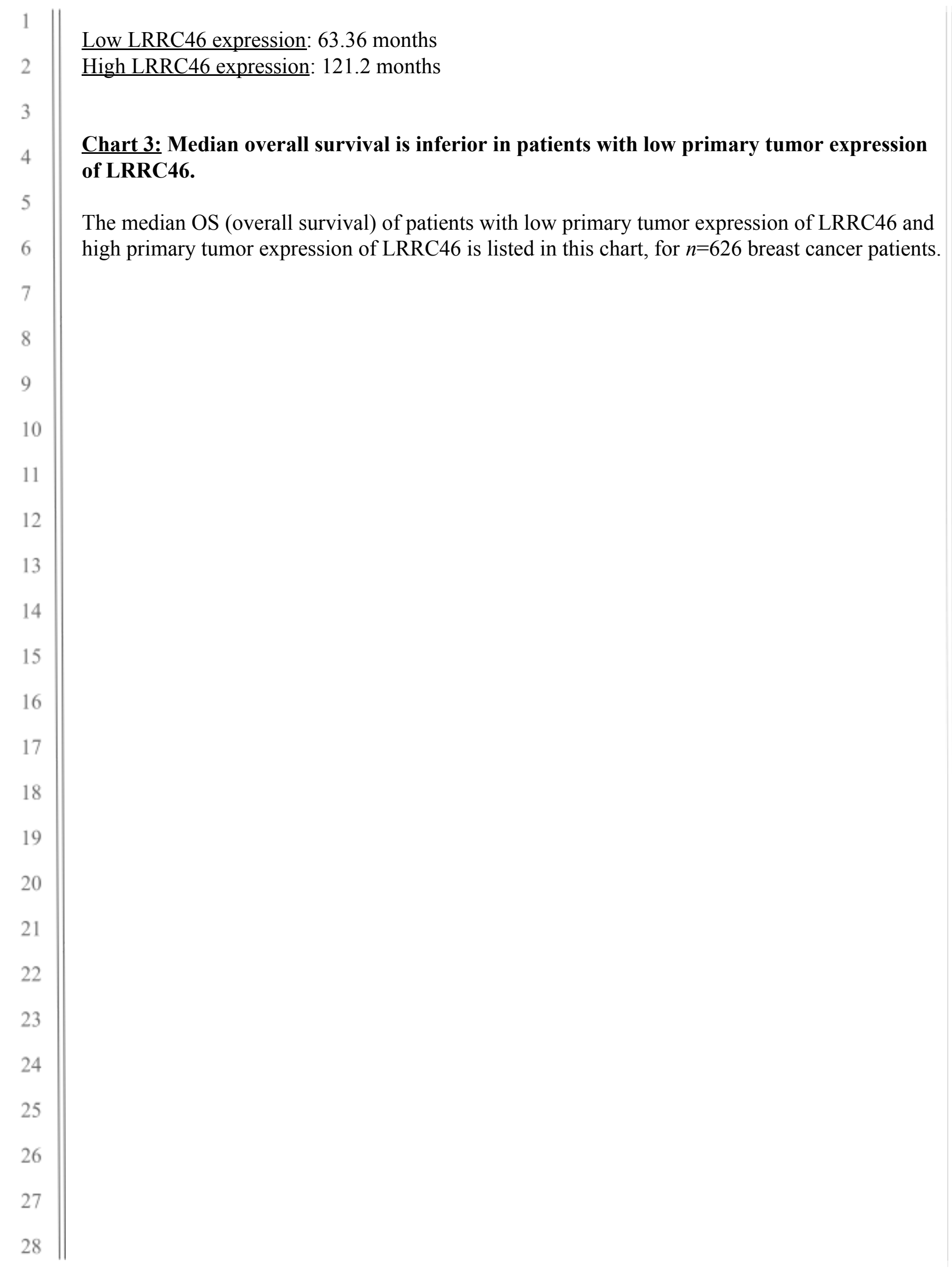




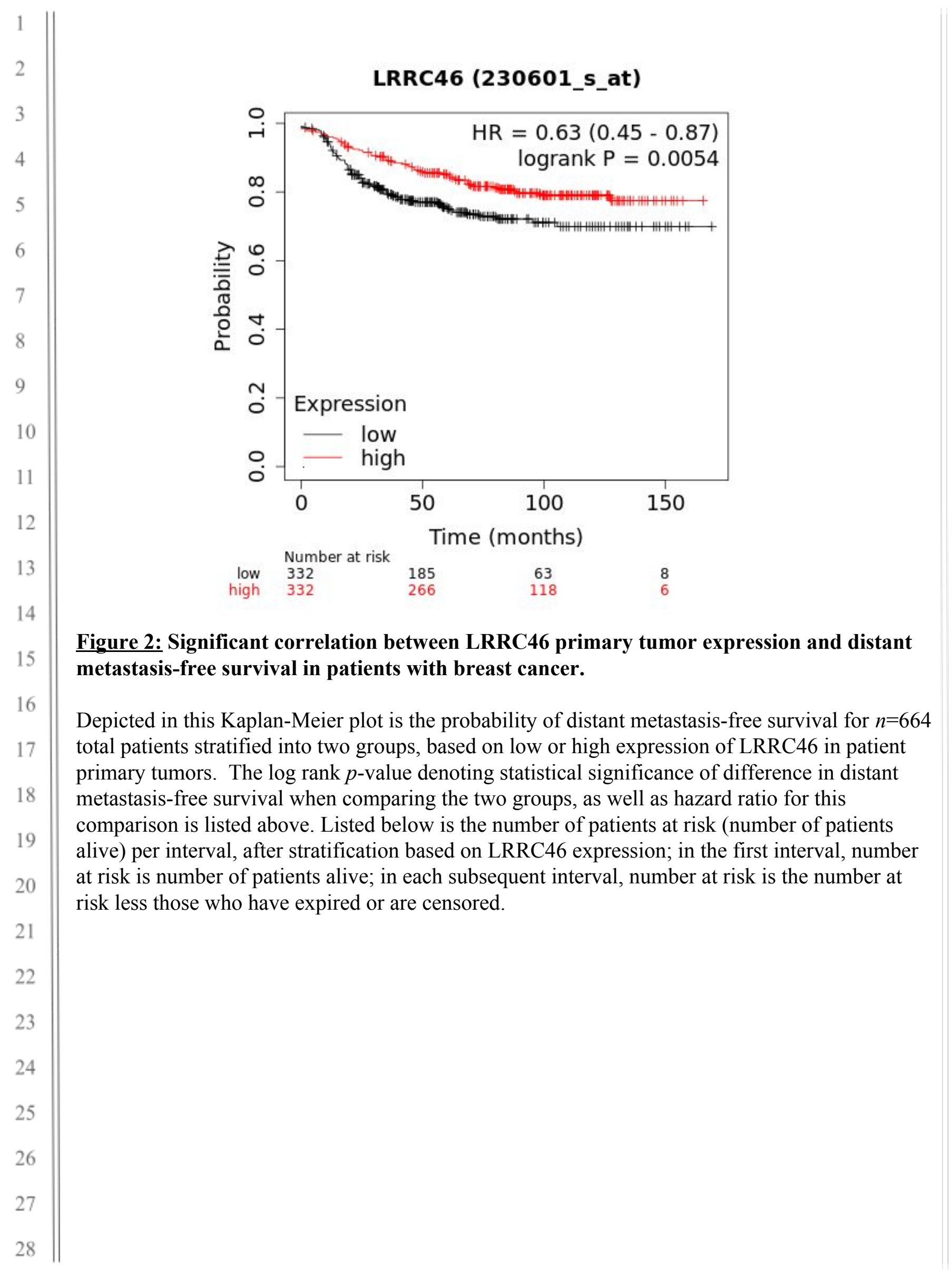

